\title{
Optimal and suboptimal control of a standard Brownian motion
}

\author{
MARIO LEFEBVRE
}

The problem of optimally controlling a standard Brownian motion until a fixed final time is considered in the case when the final cost function is an even function. Two particular problems are solved explicitly. Moreover, the best constant control as well as the best linear control are also obtained in these two particular cases.

Key words: stochastic control, Wiener process, best linear control.

\section{Introduction}

In a series of papers (see Lefebvre and Zitouni (2012) and (2014), for instance), the author considered the problem of optimally controlling a one-dimensional diffusion process until it enters a given termination set. More precisely, assume that the controlled diffusion process $\{X(t), t \geqslant 0\}$ satisfies the stochastic differential equation

$$
d X(t)=m[X(t)] d t+h[X(t)] u[X(t)] d t+\{v[X(t)]\}^{1 / 2} d B(t),
$$

in which $u(\cdot)$ is the control variable, $m(\cdot), h(\cdot)$ and $v(\cdot)>0$ are Borel measurable functions, and $\{B(t), t \geqslant 0\}$ is a standard Brownian motion. We want to find the control $u^{*}$ that minimizes the expected value of the cost function

$$
J(x)=\int_{0}^{T(x)}\left\{\frac{1}{2} q_{0} u^{2}[X(t)]+\lambda\right\} d t+K[X(T(x)), T(x)],
$$

where $q_{0}>0$ and $\lambda \neq 0$ are constants, $K$ is a general termination cost function and $T(x)$ is a random variable defined by

$$
T(x)=\inf \left\{t>0: X(t)=d_{1} \text { or } d_{2} \mid X(0)=x \in\left(x_{1}, x_{2}\right)\right\} .
$$

The Author is with Department of Mathematics and Industrial Engineering, Polytechnique Montréal, C.P. 6079, Succursale Centre-ville, Montréal, Québec H3C 3A7, Canada. Email: mlefebvre@ polymtl.ca.

This research was supported by the Natural Sciences and Engineering Research Council of Canada (NSERC).

Received 22.05.2016. 
The set of admissible controls consists of Borel measurable functions.

This type of problem, known as $L Q G$ homing (see Whittle, 1982) has also been considered recently by Makasu (2013), in which an explicit solution to a two-dimensional problem is presented.

In general, in the problems that could be solved explicitly so far, the function $K(\cdot, \cdot)$ was set equal to zero. Then, if the constant $\lambda$ is positive, the aim is to make the diffusion process leave the continuation region as soon as possible (taking the quadratic control costs into account). When $\lambda$ is negative, the optimizer tries to maximize the time spent by the controlled process in the continuation region.

In the current paper, we assume that $\{X(t), t \geqslant 0\}$ is a controlled standard Brownian motion and we replace the random variable $T(x)$ by a fixed constant $t_{f}$. Moreover, instead of giving a penalty that is proportional to the time spent by $\{X(t), t \geqslant 0\}$ in the continuation region, we assume that $\lambda$ is equal to zero and we choose the termination cost function $K$ in such a way that one is penalized if $X\left(t_{f}\right)$ is far from the objective.

In the next section, the problem set up above will be solved explicitly for two particular functions $K$. Then, in Section 3, we will consider the problem of obtaining the best constant control as well as the best linear control in the problems solved in Section 2. The expected costs obtained with these suboptimal solutions will be compared with the value function, that is, the expected cost when one uses the optimal control. Finally, a few concluding remarks will be made in Section 4.

\section{Optimal control of a standard Brownian motion}

Let $\{X(t), t \geqslant 0\}$ be the process defined by the stochastic differential equation

$$
d X(t)=b_{0} u[X(t), t] d t+d B(t),
$$

where $b_{0} \neq 0$ is a constant. The cost function is

$$
J\left(x, t_{0}\right)=\int_{t_{0}}^{t_{f}} \frac{1}{2} q_{0} u^{2}[X(t), t] d t+K\left[X\left(t_{f}\right)\right],
$$

where $x=X\left(t_{0}\right)$.

We consider an important problem known in control theory as a regulator problem. In this type of problem, the optimizer is trying to keep the controlled process as close as possible to a constant $c$, taking the quadratic control costs into account. In the case of a controlled standard Brownian motion, we can assume, without loss of generality, that $c=0$. Hence, the non-negative function $K(\cdot)$ should be such that $K\left[X\left(t_{f}\right)\right]$ is minimum when $X\left(t_{f}\right)$ is equal to zero, and should be strictly increasing with $\left|X\left(t_{f}\right)\right|$. Moreover, we assume that $K(\cdot)$ is an even function and that

$$
\lim _{X\left(t_{f}\right) \rightarrow \infty} K\left[X\left(t_{f}\right)\right]=\infty .
$$


To solve our optimal control problem, we can use dynamic programming. We define the value function

$$
F\left(x, t_{0}\right)=\inf _{u[X(t), t], t_{0} \leqslant t \leqslant t_{f}} E\left[J\left(x, t_{0}\right)\right] .
$$

This function satisfies the Hamilton-Jacobi-Bellman (HJB) equation

$$
0=\inf _{u\left(x, t_{0}\right)}\left\{\frac{1}{2} q_{0} u^{2}\left(x, t_{0}\right)+F_{t_{0}}\left(x, t_{0}\right)+b_{0} u\left(x, t_{0}\right) F_{x}\left(x, t_{0}\right)+\frac{1}{2} F_{x x}\left(x, t_{0}\right)\right\} .
$$

It follows that the optimal control $u^{*}\left(x, t_{0}\right)$ is given by

$$
u^{*}\left(x, t_{0}\right)=-\frac{b_{0}}{q_{0}} F_{x}\left(x, t_{0}\right)
$$

Substituting this expression into the HJB equation, we obtain the non-linear secondorder partial differential equation

$$
F_{t_{0}}\left(x, t_{0}\right)-\frac{b_{0}^{2}}{2 q_{0}}\left[F_{x}\left(x, t_{0}\right)\right]^{2}+\frac{1}{2} F_{x x}\left(x, t_{0}\right)=0 .
$$

Actually, $F$ also depends on $t_{f}$, and we have the boundary condition

$$
F\left(x, t_{0}\right)=K(x) \quad \text { if } t_{f}=t_{0} .
$$

We assumed above that the function $K\left[X\left(t_{f}\right)\right]$ is chosen to be strictly increasing with $\left|X\left(t_{f}\right)\right|$ and to satisfy the condition in (6). It also implies that the following conditions hold:

$$
\lim _{x \rightarrow \pm \infty} F\left(x, t_{0}\right)=\infty \text {. }
$$

Indeed, since $t_{f}$ is finite, if we start at $\pm \infty$ the value of $\left|X\left(t_{f}\right)\right|$ should also be infinite, unless the optimizer uses an infinite control $u$. At any rate, the conditions in (12) will then be satisfied.

Moreover, by symmetry, the value function $F\left(x, t_{0}\right)$ should be such that $F\left(-x, t_{0}\right)=$ $F\left(x, t_{0}\right)$. Since it must be strictly increasing with $|x|$, and $F_{x}\left(x, t_{0}\right)$ is assumed to exist, it has a minimum at $x=0$.

Next, we can greatly simplify our problem by defining

$$
G\left(x, t_{0}\right)=e^{-F\left(x, t_{0}\right) / \alpha},
$$

with

$$
\alpha:=\frac{q_{0}}{b_{0}^{2}} .
$$

Indeed, we find that the function $G$ satisfies the linear second-order partial differential equation

$$
G_{t_{0}}\left(x, t_{0}\right)+\frac{1}{2} G_{x x}\left(x, t_{0}\right)=0,
$$


subject to

$$
G\left(x, t_{0}\right)=e^{-K(x) / \alpha} \quad \text { if } t_{f}=t_{0} .
$$

Now, let $L\left(\omega, t_{0}\right)$ denote the Fourier transform with respect to the variable $x$ of the function $G\left(x, t_{0}\right)$. That is,

$$
L\left(w, t_{0}\right):=\mathcal{F}\left\{G\left(x, t_{0}\right)\right\}=\int_{-\infty}^{\infty} e^{-i w x} G\left(x, t_{0}\right) d x .
$$

Because the constant $\alpha$ is positive (and we assumed that the even function $K(\cdot)$ is such that the condition in (12) is satisfied), we can write that

$$
\lim _{x \rightarrow \pm \infty} G\left(x, t_{0}\right)=0 .
$$

Then we deduce from Eq. (15) that $L\left(\omega, t_{0}\right)$ satisfies the first-order differential equation

$$
L_{t_{0}}\left(w, t_{0}\right)-\frac{w^{2}}{2} L\left(w, t_{0}\right)=0
$$

whose general solution is

$$
L\left(w, t_{0}\right)=c_{0} e^{w^{2} t_{0} / 2},
$$

in which the constant $c_{0}$ is uniquely determined from the condition (see Eq. (16))

$$
L\left(w, t_{0}\right)=\mathcal{F}\left\{e^{-K(x) / \alpha}\right\} \quad \text { if } t_{f}=t_{0} .
$$

We may thus write that

$$
c_{0}=e^{-w^{2} t_{f} / 2} L\left(w, t_{f}\right),
$$

so that

$$
L\left(w, t_{0}\right)=e^{w^{2}\left(t_{0}-t_{f}\right) / 2} L\left(w, t_{f}\right)=e^{w^{2}\left(t_{0}-t_{f}\right) / 2} \mathcal{F}\left\{e^{-K(x) / \alpha}\right\} .
$$

We can now state the following result.

Proposition 1 If the even function $K$ is chosen in such a way that the condition in (6) is satisfied, then the optimal control $u^{*}\left(x, t_{0}\right)$ is given by

$$
u^{*}\left(x, t_{0}\right)=-\alpha \frac{b_{0}}{q_{0}} \frac{G_{x}\left(x, t_{0}\right)}{G\left(x, t_{0}\right)}=-\frac{1}{b_{0}} \frac{\frac{d}{d x} \mathcal{F}^{-1}\left\{L\left(w, t_{0}\right)\right\}}{\mathcal{F}^{-1}\left\{L\left(w, t_{0}\right)\right\}} .
$$

Moreover, the value function is

$$
F\left(x, t_{0}\right)=-\frac{q_{0}}{b_{0}^{2}} \ln \left\{\mathcal{F}^{-1}\left\{e^{w^{2}\left(t_{0}-t_{f}\right) / 2} L\left(w, t_{f}\right)\right\}\right\} .
$$




\subsection{Particular cases}

I) The first particular case that we consider is the one for which

$$
K\left[X\left(t_{f}\right)\right]=X^{2}\left(t_{f}\right) .
$$

Furthermore, we set $b_{0}=q_{0}=1$, so that $\alpha=1$.

Remark. Because the control costs are also quadratic, the optimal control $u^{*}\left(x, t_{0}\right)$ should be a linear function of $x$.

With this choice for the even function $K$, we have

$$
\lim _{X\left(t_{f}\right) \rightarrow \infty} K\left[X\left(t_{f}\right)\right]=\infty
$$

as required, so that we can use Proposition 1. We have

$$
L\left(w, t_{f}\right)=\mathcal{F}\left\{e^{-x^{2}}\right\}=\sqrt{\pi} e^{-w^{2} / 4} .
$$

It follows that

$$
L\left(w, t_{0}\right)=e^{w^{2}\left(t_{0}-t_{f}\right) / 2} \sqrt{\pi} e^{-w^{2} / 4}
$$

We find that

$$
G\left(x, t_{0}\right)=\mathcal{F}^{-1}\left\{L\left(w, t_{0}\right)\right\}=\frac{1}{\sqrt{1+2\left(t_{f}-t_{0}\right)}} \exp \left\{-\frac{x^{2}}{2\left(t_{f}-t_{0}\right)+1}\right\},
$$

which implies that the value function is given by

$$
F\left(x, t_{0}\right)=\frac{1}{2} \ln \left[1+2\left(t_{f}-t_{0}\right)\right]+\frac{x^{2}}{2\left(t_{f}-t_{0}\right)+1}
$$

and the optimal control is

$$
u^{*}\left(x, t_{0}\right)=-F_{x}\left(x, t_{0}\right)=-\frac{2 x}{2\left(t_{f}-t_{0}\right)+1} .
$$

Remarks. (i) We see that the optimal control is indeed a linear function of $x$, as expected. (ii) The function $F\left(x, t_{0}\right)$ is symmetrical with respect to 0 and has a minimum at the origin, as it should.

II) We now choose the following termination cost function:

$$
K\left[X\left(t_{f}\right)\right]=\left|X\left(t_{f}\right)\right|
$$


and we still take $b_{0}=q_{0}=1$. Again, we can appeal to Proposition 1. This time, we find that

$$
L\left(w, t_{f}\right)=\mathcal{F}\left\{e^{-|x|}\right\}=\frac{2}{1+w^{2}},
$$

so that

$$
L\left(w, t_{0}\right)=e^{w^{2}\left(t_{0}-t_{f}\right) / 2} \frac{2}{1+w^{2}} .
$$

We can invert the Fourier transform to obtain the function $G$ :

$$
\begin{aligned}
G\left(x, t_{0}\right)= & \frac{1}{2} e^{\left(t_{f}-t_{0}\right) / 2}\left\{e^{-x} \operatorname{erf}\left(\frac{x+t_{0}-t_{f}}{\sqrt{2\left(t_{f}-t_{0}\right)}}\right)+2 \cosh (x)\right. \\
& \left.-e^{x} \operatorname{erf}\left(\frac{x+t_{f}-t_{0}}{\sqrt{2\left(t_{f}-t_{0}\right)}}\right)\right\},
\end{aligned}
$$

where erf denotes the error function. From this expression for $G$, it is a simple matter to calcule the value function

$$
\begin{aligned}
F\left(x, t_{0}\right)= & -\ln (2)-\frac{t_{f}-t_{0}}{2}-\ln \left\{e^{-x} \operatorname{erf}\left(\frac{x+t_{0}-t_{f}}{\sqrt{2\left(t_{f}-t_{0}\right)}}\right)+2 \cosh (x)\right. \\
& \left.-e^{x} \operatorname{erf}\left(\frac{x+t_{f}-t_{0}}{\sqrt{2\left(t_{f}-t_{0}\right)}}\right)\right\}
\end{aligned}
$$

and the optimal control

$$
u^{*}\left(x, t_{0}\right)=-\frac{\operatorname{erf}\left(\frac{x+t_{0}-t_{f}}{\sqrt{2\left(t_{f}-t_{0}\right)}}\right) e^{-x}-2 \sinh (x)+\operatorname{erf}\left(\frac{x+t_{f}-t_{0}}{\sqrt{2\left(t_{f}-t_{0}\right)}}\right) e^{x}}{\operatorname{erf}\left(\frac{x+t_{0}-t_{f}}{\sqrt{2\left(t_{f}-t_{0}\right)}}\right) e^{-x}+2 \cosh (x)-\operatorname{erf}\left(\frac{x+t_{f}-t_{0}}{\sqrt{2\left(t_{f}-t_{0}\right)}}\right) e^{x}} .
$$

We can check that the value function is symmetrical with respect to 0 and has a minimum at the origin. It follows that $u^{*}\left(0, t_{0}\right)=0$, which is logical.

We see that a seemingly almost equivalent problem leads to a much more complicated optimal solution. In the next section, we will compute the best linear control for this particular problem. We will also compute the best constant control for both particular cases presented above, as well as the expected cost when the optimizer uses no control at all. The various expected costs obtained by using all these suboptimal controls will then be compared to the value function in special instances.

\section{Suboptimal solutions to our problems}

First, we will compute the expected cost when the optimizer uses no control at all, so that $u[X(t), t] \equiv 0$. Denoting the controlled process in this case by $\left\{X_{0}(t), t \geqslant t_{0}\right\}$ and 
the cost function by $J_{0}\left(x, t_{0}\right)$, we have

$$
E\left[J_{0}\left(x, t_{0}\right)\right]=E\left[K\left[X_{0}\left(t_{f}\right)\right]\right] .
$$

Moreover, as is well known, $X_{0}\left(t_{f}\right)$ has a Gaussian distribution with mean $X_{0}\left(t_{0}\right)=x$ and variance $t_{f}-t_{0}$. It follows that

$$
E\left[X_{0}^{2}\left(t_{f}\right)\right]=t_{f}-t_{0}+x^{2}
$$

Now, if $Z \sim \mathrm{N}\left(\mu, \sigma^{2}\right)$, we find that

$$
E[|Z|]=-\mu+\sqrt{\frac{2}{\pi}} \sigma \exp \left\{-\frac{\mu^{2}}{2 \sigma^{2}}\right\}+2 \mu \Phi(\mu / \sigma),
$$

where $\Phi$ denotes the distribution function of the $\mathrm{N}(0,1)$ random variable. Making use of this formula, we obtain that

$$
E\left[\left|X_{0}\left(t_{f}\right)\right|\right]=-x+\sqrt{\frac{2\left(t_{f}-t_{0}\right)}{\pi}} \exp \left\{-\frac{x^{2}}{2\left(t_{f}-t_{0}\right)}\right\}+2 x \Phi\left(\frac{x}{\sqrt{t_{f}-t_{0}}}\right) .
$$

Next, we will compute the best constant control for the two problems considered in the previous section. If $u[X(t), t] \equiv c$, denoting the controlled process by $X_{c}(t)$, we have

$$
d X_{c}(t)=c d t+d B(t)
$$

Then $\left\{X_{c}(t), t \geqslant t_{0}\right\}$ is a Wiener process with drift $c$ and variance parameter 1 . It follows that, starting from $X_{c}\left(t_{0}\right)=x$,

$$
X_{c}(t) \sim \mathrm{N}\left(x+c\left(t_{f}-t_{0}\right), t_{f}-t_{0}\right) .
$$

Hence, we may write that the expected value of the cost function (which we denote by $J_{c}\left(x, t_{0}\right)$ when $\left.u[X(t), t] \equiv c\right)$ is given by

$$
E\left[J_{c}\left(x, t_{0}\right)\right]=\frac{1}{2} c^{2}\left(t_{f}-t_{0}\right)+E\left[K\left[X_{c}\left(t_{f}\right)\right]\right] .
$$

Thus, if $K\left[X_{c}\left(t_{f}\right)\right]=X_{c}^{2}\left(t_{f}\right)$,

$$
E\left[J_{c}\left(x, t_{0}\right)\right]=\frac{1}{2} c^{2}\left(t_{f}-t_{0}\right)+\left(t_{f}-t_{0}\right)+\left[x+c\left(t_{f}-t_{0}\right)\right]^{2},
$$

while in the case when $K\left[X_{c}\left(t_{f}\right)\right]=\left|X_{c}\left(t_{f}\right)\right|$, we obtain (see Eq. (40)) that

$$
\begin{aligned}
E\left[J_{c}\left(x, t_{0}\right)\right]= & \frac{1}{2} c^{2}\left(t_{f}-t_{0}\right)-x-c\left(t_{f}-t_{0}\right) \\
& +\sqrt{\frac{2\left(t_{f}-t_{0}\right)}{\pi}} \exp \left\{-\frac{\left[x+c\left(t_{f}-t_{0}\right)\right]^{2}}{2\left(t_{f}-t_{0}\right)}\right\}
\end{aligned}
$$




$$
+2\left[x+c\left(t_{f}-t_{0}\right)\right] \Phi\left(\frac{x+c\left(t_{f}-t_{0}\right)}{\sqrt{t_{f}-t_{0}}}\right) .
$$

The best constant control is determined by finding the value of $c$ that minimizes $E\left[J_{c}\left(x, t_{0}\right)\right]$. In the first case, we have

$$
\frac{d}{d c} E\left[J_{c}\left(x, t_{0}\right)\right]=c\left(t_{f}-t_{0}\right)+2\left[x+c\left(t_{f}-t_{0}\right)\right]\left(t_{f}-t_{0}\right),
$$

so that the best constant $c^{*}$ is given by

$$
c^{*}=-\frac{2 x}{1+2\left(t_{f}-t_{0}\right)} .
$$

The expected cost becomes

$$
E\left[J_{c^{*}}\left(x, t_{0}\right)\right]=\frac{x^{2}}{1+2\left(t_{f}-t_{0}\right)}+t_{f}-t_{0} .
$$

Remarks. (i) We can check that this value of the constant $c$ corresponds indeed to a minimum for the expected cost. (ii) We see that if we start at $X_{c}\left(t_{0}\right)=0$, then the best constant is $c^{*}=0$, so that $E\left[J_{c^{*}}\left(x, t_{0}\right)\right]$ reduces to $E\left[J_{0}\left(x, t_{0}\right)\right]$. (iii) The value of $c^{*}$ depends on $x$. However, the control will not change between $t_{0}$ and $t_{f}$; that is, $u[X(t), t]=c^{*}$ for all values of $t \in\left[t_{0}, t_{f}\right]$.

Now, writing

$$
\Delta t:=t_{f}-t_{0},
$$

we find that the optimal constant $c$ when $K\left[X_{c}\left(t_{f}\right)\right]=\left|X_{c}\left(t_{f}\right)\right|$ is such that (after simplification)

$$
0=\frac{d}{d c} E\left[J_{c}\left(x, t_{0}\right)\right]=c \Delta t-\Delta t+2 \Delta t \Phi\left(\frac{x+c \Delta t}{\sqrt{\Delta t}}\right) .
$$

Hence, $c^{*}$ satisfies the equation

$$
c^{*}-1+2 \Phi\left(\frac{x+c^{*} \Delta t}{\sqrt{\Delta t}}\right)=0 .
$$

Using a mathematical software, this equation can be solved for any $x$ and $\Delta t$, which then enables us to compute the expected cost explicitly. A numerical example will be provided at the end of this section.

The most difficult task is to determine the best linear control when $K\left[X_{c}\left(t_{f}\right)\right]=$ $\left|X_{c}\left(t_{f}\right)\right|$. Since we were able to obtain the exact optimal control $u^{*}\left(x, t_{0}\right)$ in this case, we could try to find a linear approximation to $u^{*}\left(x, t_{0}\right)$. However, here we can find the 
exact constant $a$ such that $u_{a}[X(t), t]:=a X_{a}(t)$ minimizes the expected value of the cost function

$$
J_{a}\left(x, t_{0}\right):=\int_{t_{0}}^{t_{f}} \frac{1}{2} a^{2} X_{a}^{2}(t) d t+\left|X_{a}\left(t_{f}\right)\right|,
$$

with $\left\{X_{a}(t), t \geqslant t_{0}\right\}$ defined by

$$
d X_{a}(t)=a X_{a}(t) d t+d B(t) .
$$

Since the aim is to bring the controlled process as close as possible to zero at time $t_{f}$, we can assume that the optimal constant $a$ is negative. Let $b:=-a$. The process $\left\{X_{b}(t), t \geqslant t_{0}\right\}$ that satisfies the stochastic differential equation

$$
d X_{b}(t)=-b X_{b}(t) d t+d B(t)
$$

is an Ornstein-Uhlenbeck process, which is a time-homogeneous Gaussian process. We can write (see Cox and Miller (1965), for instance) that, conditional on $X_{b}\left(t_{0}\right)=x$,

$$
X_{b}(t) \sim \mathrm{N}\left(x e^{-b\left(t_{f}-t_{0}\right)}, \frac{1-e^{-2 b\left(t_{f}-t_{0}\right)}}{2 b}\right) .
$$

It follows (with $\Delta t=t_{f}-t_{0}$, as above) that

$$
E\left[X_{b}^{2}(t)\right]=\frac{1-e^{-2 b \Delta t}}{2 b}+\left(x e^{-b \Delta t}\right)^{2} .
$$

Hence, the expected cost when we choose $u[X(t), t]=u_{b}[X(t), t]:=-b X_{b}(t)$ is given by

$$
\begin{aligned}
E\left[J_{b}\left(x, t_{0}\right)\right] & =\int_{t_{0}}^{t_{f}} \frac{1}{2} b^{2} E\left[X_{b}^{2}(t)\right] d t+E\left[\left|X_{b}\left(t_{f}\right)\right|\right] \\
& =\frac{b \Delta t}{4}-\frac{1}{4}\left(\frac{1}{2}-b x^{2}\right)\left(1-e^{-2 b \Delta t}\right)+E\left[\left|X_{b}\left(t_{f}\right)\right|\right],
\end{aligned}
$$

where

$$
\begin{aligned}
E\left[\left|X_{b}(t)\right|\right]= & -x e^{-b \Delta t}+\sqrt{\frac{1-e^{-2 b \Delta t}}{b \pi}} \exp \left\{-b x^{2} \frac{e^{-2 b \Delta t}}{1-e^{-2 b \Delta t}}\right\} \\
& +2 x e^{-b \Delta t} \Phi\left(\sqrt{2 b} x \frac{e^{-b \Delta t}}{\sqrt{1-e^{-2 b \Delta t}}}\right) .
\end{aligned}
$$

To obtain the optimal constant $b^{*}$, we must therefore differentiate the expected cost with respect to $b$ and use a mathematical software to find the value of $b$ for which the derivative is equal to zero (and check that this value corresponds indeed to a minimum, which clearly should be the case). Once $b^{*}$ has been evaluated (approximately), we can compute the minimum expected cost. We will illustrate the procedure with a numerical example in the next subsection. 


\subsection{A numerical application}

We will compute the various optimal and suboptimal controls derived in the previous sections, as well as the corresponding expected costs, in two particular instances. We set $t_{0}=0$ and $t_{f}=1$, so that $\Delta t=1$, and we first assume that the controlled process starts at $x=1$.

I) In the first example considered in Section 2, if the optimizer uses no control at all, then the expected cost is given by (see Eq. (39)) $E\left[J_{0}(1,0)\right]=2$. Moreover, the best constant control $c^{*}$ is (see Eq. (48)) $=-2 / 3$, and the corresponding expected cost (see Eq. (49)) is equal to $5 / 3$. Finally, we deduce from (31) and (30), respectively, that the optimal control is $u^{*}(1,0)=-2 x / 3$ and the value function is

$$
F(1,0)=\frac{1}{2} \ln (3)+\frac{1}{3} \simeq 0,88 .
$$

We can conclude that, for this numerical example, the optimizer should use some control. Furthermore, the optimal solution is much better than the best constant control.

If we replace the final time $t_{f}=1$ by $t_{f}=10$, we obtain the following results: $E\left[J_{0}(1,0)\right]=11, c^{*}=-2 / 21, E\left[J_{c^{*}}(1,0)\right] \simeq 10,05, u^{*}(1,0)=-2 x / 21$ and $F(1,0) \simeq$ 1,57 . We see that for $t_{f}$ large, there is a huge difference between the expected cost obtained with the best constant control and the value function.

II) When $K\left[X_{c}\left(t_{f}\right)\right]=\left|X_{c}\left(t_{f}\right)\right|$, if $t_{0}=0, t_{f}=1$ and $x=1$, we find (see Eq. (41)) that

$$
E\left[J_{0}(1,0)\right]=E\left[\left|X_{0}(1)\right|\right]=-1+\sqrt{2 / \pi} e^{-1 / 2}+2 \Phi(1) \simeq 1,17 .
$$

The best constant $c^{*}$ satisfies Eq. (52), which becomes

$$
c^{*}-1+2 \Phi\left(1+c^{*}\right)=0 .
$$

We find that $c^{*} \simeq 0,43$. Making use of this value, we deduce from Eq. (46) that $E\left[J_{c^{*}}(1,0)\right] \simeq 1,02$.

Next, with the help of a mathematical software, we obtain that $b^{*} \simeq 0,56$. Hence, we compute (see (58) and (59)) that $E\left[J_{b^{*}}(1,0)\right] \simeq 0,93$.

Finally, we find that the value function $F(1,0)$ is approximately equal to 0,90 . Therefore, here there is less difference between the optimal and suboptimal solutions, compared with the first example presented above.

As in the previous particular case, we replace $t_{f}=1$ by $t_{f}=10$ to see whether the suboptimal solutions are still close to the optimal one when $t_{f}$ is large. We find that $E\left[J_{0}(1,0)\right] \simeq 2,65, c^{*} \simeq-0,072, E\left[J_{c^{*}}(1,0)\right] \simeq 2,56, b^{*} \simeq 0,63, E\left[J_{b^{*}}(1,0)\right] \simeq 2,31$ and $F(1,0) \simeq 1,50$. Although the suboptimal expected costs are closer to the value function than in the first example with $t_{f}=10$, it is clear that the optimal control $u^{*}(1,0)$ is really superior to the suboptimal controls. 


\section{Conclusion}

In this paper, the problem of optimally controlling a standard Brownian motion until a fixed time $t_{f}$ when the termination cost function $K$ is an even function has been considered. In Section 2, we saw that if we are able to invert the Fourier transform $L\left(w, t_{0}\right)$ defined in (17), then we obtain explicit expressions for the optimal control and the value function.

The Fourier transform $L$ depends on the choice of the termination cost function $K$. In Section 2, we presented the exact solution to our optimal control problem when $K\left[X\left(t_{f}\right)\right]=X^{2}\left(t_{f}\right)$ and when $K\left[X\left(t_{f}\right)\right]=\left|X\left(t_{f}\right)\right|$. As expected, $u^{*}\left(x, t_{0}\right)$ is linear in $x$ in the first case. However, the optimal solution was not obvious at all when we replace the square of the final position $X\left(t_{f}\right)$ of the process by its absolute value.

In Section 3, we computed various suboptimal solutions to the problem set up in this paper. The numerical examples presented at the end of this section showed that these suboptimal solutions could not compete with the optimal one, at least for the numerical values chosen for $t_{0}, t_{f}$ and $x$. Nevertheless, in the case when we are unable to invert the Fourier transform $L\left(w, t_{0}\right)$, the suboptimal solutions are worth considering, especially the best linear control.

We could have considered a controlled Wiener process with drift $\mu$ and variance parameter $\sigma^{2}$ instead of a standard Brownian motion. It would be interesting to see the effect of the variance parameter on the optimal solution. However, we could then no longer use symmetry to assert that $F\left(-x, t_{0}\right)=F\left(x, t_{0}\right)$.

Finally, we could try to extend the results presented in this paper by replacing the deterministic final time $t_{f}$ by a random variable $T$ that is independent of the controlled process $\left\{X(t), t \geqslant t_{0}\right\}$. We could also consider the case when the infinitesimal parameters of the controlled process are not both constants, but at least one is rather a function of $X(t)$ and/or $t$. For instance, we could assume that $\left\{X(t), t \geqslant t_{0}\right\}$ is an Ornstein-Uhlenbeck process, whose infinitesimal mean is given by $-\beta x$ for a positive constant $\beta$.

\section{References}

[1] D.R.Cox and H.D. MilleR: The Theory of Stochastic Processes. Methuen: London; 1965.

[2] M. LeFEB VRE and F. ZITOUNI: General LQG homing problems in one dimension. Int. J. Stoch. Anal., Article ID 803724, (2012), doi:10.1155/2012/803724.

[3] M. Lefeb VRe and F. ZITouni: Analytical solutions to LQG homing problems in one dimension. Systems Science and Control Engineering, An Open Access Journal, 2 (2014), 41-47.

[4] C. MAKASU: Explicit solution for a vector-valued LQG homing problem. Optim. Lett., 7 (2013), 607-612. 
[5] P. Whittle: Optimization over Time, Vol. I. Chichester: Wiley; 1982. 